\title{
The topological cyclic Deligne conjecture
}

\author{
PAOLO SALVATORE
}

\begin{abstract}
Let $O$ be a cyclic topological operad with multiplication. In the framework of the cosimplicial machinery by McClure and Smith, we prove that the totalization of the cosimplicial space associated to $O$ has an action of an operad equivalent to the framed little 2-discs operad.
\end{abstract}

$18 \mathrm{D} 50 ; 55 \mathrm{P} 48$

\section{Introduction}

The Deligne conjecture, proved by several authors, states that the little 2-discs operad $\mathcal{D}_{2}$ acts on the chain level on the Hochschild complex of any associative algebra. The question was motivated by a well known action on the homological level (Gerstenhaber [5]). The natural generalization of the conjecture is about the Hochschild complex of an operad with multiplication, that is an operad with a map from the associative operad. The original statement is recovered taking the endomorphism operad of an associative algebra. McClure and Smith proved a topological version of the Deligne conjecture $[17 ; 18 ; 19]$, where the Hochschild complex is replaced by the totalization of a cosimplicial space associated to a topological operad with multiplication. A major example, constructed by Sinha [24], occurs when the operad with multiplication is a version of the little $n$-discs operad, the Kontsevich operad $\mathcal{K}_{n}$. The (homotopy) totalization of $\mathcal{K}_{n}$ has as factor the space $\mathrm{Emb}_{n}$ of long knots in $\mathbb{R}^{n}$ for $n>3$. We proved in [22] that $\mathrm{Emb}_{n}$ itself has an action of the little 2-discs operad. The cyclic Deligne conjecture states that the operad of framed little 2-discs $f \mathcal{D}_{2}$ acts on the chain level on the Hochschild complex of a Frobenius algebra. The conjecture was proved in this form by Kaufmann [11], and Kontsevich-Soibelman [14]. Since the endomorphism operad of a Frobenius algebra is cyclic, the natural generalization of the conjecture claims an action of the chains of $f \mathcal{D}_{2}$ on the Hochschild complex of a cyclic operad with multiplication. This statement was proved on the homological level by Menichi [20]. The topological counterpart of the statement says that the framed little 2-discs operad $f \mathcal{D}_{2}$ acts on the totalization of a topological cyclic operad with multiplication. This is the main result of our paper (Theorem 7.7). An equivalent 
statement was conjectured by McClure and Smith [19]. Hu [8] stated a related result and applied it to the Hochschild complex of commutative Frobenius algebras.

We will revisit the proof by McClure and Smith of the non-cyclic statement showing that the model of $\mathcal{D}_{2}$ they used, denoted here $M S$, is strongly related to Kaufmann's spineless cacti operad. In fact the elements of $M S$ can be thought of as spineless cacti together with a monotone parametrization. As far as we know this has not been observed before. The parametrizations are necessary if we want to include the constant base point at the 0 level of the operad.

We will then construct an operad $f M S$, a model of $f \mathcal{D}_{2}$, whose elements correspond to Voronov's cacti with a parametrization, and prove the main theorem. The proof is conceptually similar to that of the non-cyclic statement, except that the triple defining cyclic operads rather than non-cyclic operads comes into play.

The main motivation of this paper is a joint project of Ryan Budney and the author to endow the space $f \mathrm{Emb}_{n}$ of framed long knots in $\mathbb{R}^{n}$ with a meaningful action of the framed little 2-discs operad. The action is expected because $f \mathrm{Emb}_{n}$ is the (homotopy) totalization of an operad with multiplication equivalent to the framed little $n$-discs operad $f \mathcal{D}_{n}$ [22], and $f \mathcal{D}_{n}$ is equivalent to a cyclic operad [2].

The paper is relatively self-contained. In Section 2 and Section 3 we recall the definitions and give several examples of cosimplicial spaces and (non-symmetric) operads. In Section 4 we define geometrically the operad $M S$ and its framed version $f M S$. Then we reprove in Section 5 the theorem of McClure and Smith (Theorem 5.4) from this geometric point of view. In Section 6 we recall the definition of the cyclic category and of a cocyclic space. In Section 7 we define cyclic operads, and we prove the main Theorem 7.7. For example the theorem gives an action of $f M S$ on based loop groups. Another important example we recover is the string topology action of $f M S$ on a free loop space, that makes sense according to Voronov [25] in the category of correspondences. This paper grew out of my lecture notes at the Lille Summer School on Algebraic Topology in June 2007. I am grateful to the Laboratoire Painlevé and in particular to Sadok Kallel for support and hospitality.

\section{Cosimplicial spaces}

We recall the definition of a cosimplicial space. Let $\Delta$ be the category with the standard ordered sets $[k]=\{0<\cdots<k\}$ as objects $(k \in \mathbb{N})$ and monotone maps as morphisms. For a fixed $n \in \mathbb{N}$ and $0 \leq i \leq n+1$ let $\delta_{i}:[n] \rightarrow[n+1]$ be the morphism such that $\delta_{i}(j)=j$ if $j<i$ and $\delta_{i}(j)=j+1$ if $j \geq i$. 
For $0 \leq i \leq n$ let $\sigma_{i}:[n+1] \rightarrow[n]$ be the morphism such that $\sigma_{i}(j)=j$ if $j \leq i$ and $\sigma_{i}(j)=j-1$ if $j>i$. It is well known that $\Delta$ is the category generated by these morphisms under the relations

$$
\begin{aligned}
\delta_{j} \delta_{i} & =\delta_{i} \delta_{j-1} \quad i<j \\
\sigma_{j} \sigma_{i} & =\sigma_{i} \sigma_{j+1} \quad i \leq j \\
\sigma_{j} \delta_{i} & =\delta_{i} \sigma_{j-1} \quad i<j \\
\sigma_{j} \delta_{i} & =\mathrm{id} \quad j \leq i \leq j+1 \\
\sigma_{j} \delta_{i} & =\delta_{i-1} \sigma_{j} \quad i>j+1 .
\end{aligned}
$$

Definition 2.1 A cosimplicial space is a covariant functor from the category $\Delta$ to the category of topological spaces. The image under such functor of a morphism $\delta_{i}$ is a coface denoted $d^{i}$ and the image of a morphism $\sigma_{j}$ is a codegeneracy denoted $s^{j}$.

The definition is dual to that of a simplicial space, that is a contravariant functor from $\Delta$ to the category of topological spaces.

Example 2.2 Let us consider the $k$-simplex

$$
\Delta^{k}=\left\{\left(x_{1}, \ldots, x_{k}\right) \quad \mid 0 \leq x_{1} \leq \cdots \leq x_{k} \leq 1\right\} .
$$

The collection $\Delta^{*}$ forms a cosimplicial space with the cofaces

$$
\begin{aligned}
d^{i}\left(x_{1}, \ldots, x_{k}\right) & =\left(x_{1}, \ldots, x_{i}, x_{i}, \ldots, x_{k}\right) \quad 1 \leq i \leq k \\
d^{0}\left(x_{1}, \ldots, x_{k}\right) & =\left(0, x_{1}, \ldots, x_{k}\right) \\
d^{k+1}\left(x_{1}, \ldots, x_{k}\right) & =\left(x_{1}, \ldots, x_{k}, 1\right)
\end{aligned}
$$

and with the codegeneracies

$$
s^{i}\left(x_{1}, \ldots, x_{k}\right)=\left(x_{1}, \ldots, \widehat{x_{i+1}}, \ldots, x_{k}\right) \quad 0 \leq i \leq k-1 .
$$

Definition 2.3 The totalization $\operatorname{Tot}\left(S^{*}\right)$ of a cosimplicial space $S^{*}$ is the space of natural transformations of functors $\Delta^{*} \rightarrow S^{*}$. This means that

$$
\operatorname{Tot}\left(S^{*}\right) \subset \prod_{k \in \mathbb{N}} \operatorname{Map}\left(\Delta^{k}, S^{k}\right)
$$

is the space of sequences of maps $f^{k}: \Delta^{k} \rightarrow S^{k}$ commuting with the codegeneracies and the cofaces. 
The definition is dual to that of the realization of a simplicial space.

We recall two standard examples of cosimplicial spaces.

Example 2.4 (The cosimplicial model for the based loop space $\Omega X$ ) For a given topological space $X$ with base point $* \in X$ let us consider the cosimplicial space $\omega X$ such that $(\omega X)^{k}=X^{k}$, with

$$
\begin{aligned}
& d^{0}\left(x_{1}, \ldots, x_{k}\right)=\left(*, x_{1}, \ldots, x_{k}\right) \\
& d^{i}\left(x_{1}, \ldots, x_{k}\right)=\left(x_{1}, \ldots, x_{i}, x_{i}, \ldots x_{k}\right) \quad 1 \leq i \leq k \\
& d^{k+1}\left(x_{1}, \ldots, x_{k}\right)=\left(x_{1}, \ldots, x_{k}, *\right) \\
& s^{i}\left(x_{1}, \ldots, x_{k+1}\right)=\left(x_{1}, \ldots, \widehat{x_{i+1}}, \ldots, x_{k+1}\right) \quad 0 \leq i \leq k .
\end{aligned}
$$

It is well known that the evaluation maps $\Omega X \times \Delta^{k} \rightarrow X^{k}$

$$
\left(\gamma,\left(x_{1}, \ldots, x_{k}\right)\right) \mapsto\left(\gamma\left(x_{1}\right), \ldots, \gamma\left(x_{k}\right)\right)
$$

induce by adjointness a homeomorphism $\Omega X \cong \operatorname{Tot}(\omega X)$.

Example 2.5 (The cosimplicial model for the free loop space $L X$ ) For a topological space $X$, let us consider the cosimplicial space $l X$ such that

$$
\begin{aligned}
& (l X)^{k}=X^{k+1}=\left\{\left(x_{0}, \ldots, x_{k}\right) \mid x_{i} \in X\right\} \\
& d^{i}\left(x_{0}, \ldots, x_{k}\right)=\left(x_{0}, \ldots, x_{i}, x_{i}, \ldots, x_{k}\right) \quad 0 \leq i \leq k \\
& d^{k+1}\left(x_{0}, \ldots, x_{k}\right)=\left(x_{0}, \ldots, x_{k}, x_{0}\right) \\
& s^{i}\left(x_{0}, \ldots, x_{k+1}\right)=\left(x_{0}, \ldots, \widehat{x_{i+1}}, \ldots, x_{k+1}\right) \quad 0 \leq i \leq k .
\end{aligned}
$$

The evaluation map $L X \times \Delta^{k} \rightarrow X^{k+1}$ sending

$$
\left(\gamma,\left(x_{1}, \ldots, x_{k}\right)\right) \mapsto\left(\gamma(0), \gamma\left(x_{1}\right), \ldots, \gamma\left(x_{k}\right)\right)
$$

induces by adjointness a homeomorphism $L X \cong \operatorname{Tot}(l X)$. Actually, modulo this homeomorphism, the cosimplicial inclusion $i: \omega X \rightarrow l X$ defined by $i^{k}\left(x_{1}, \ldots, x_{k}\right)=$ $\left(*, x_{1}, \ldots, x_{k}\right)$ corresponds to the inclusion $\Omega X \rightarrow L X$.

\section{Operads}

We recall some material on operads. For more details we refer to Markl-ShniderStasheff [15]. Roughly speaking an element of an operad is an operation with many inputs and one output. 
Definition 3.1 A non-symmetric topological operad $O$ is a collection of spaces $O(k), k \in \mathbb{N}$, together with composition maps

$$
\circ_{t}: O(k) \times O(l) \rightarrow O(k+l-1) \quad 1 \leq t \leq k
$$

such that for $a \in O(n), b \in O(p), c \in O(q)$

$$
\begin{array}{rlrl}
\left(a \circ_{i} b\right) \circ_{j+p-1} c & =\left(a \circ_{j} c\right) \circ_{i} b & 1 \leq i<j \leq n \\
a \circ_{i}\left(b \circ_{j} c\right) & =\left(a \circ_{i} b\right) \circ_{i+j-1} c & 1 \leq i \leq n, 1 \leq j \leq p .
\end{array}
$$

In addition there is a point $\iota \in O(1)$, the unit, that is a bi-sided unit for the $o_{t}$-operations.

The operad is symmetric if for each natural number $k$ the symmetric group $\Sigma_{k}$ acts on $O(k)$ on the right, compatibly with the composition maps [15], in the sense that for $x \in O(m), y \in O(n), \sigma \in \Sigma_{m}$ and $\tau \in \Sigma_{n}$

$$
(x \sigma) \circ_{i}(y \tau)=\left(x \circ_{i} y\right)\left(\sigma \circ_{i} \tau\right)
$$

where $\sigma \circ_{i} \tau \in \Sigma_{m+n-1}$ is the permutation exchanging by $\sigma m$ consecutive blocks having all one element except the $i$ th having $n$ elements, and acting by $\tau$ on the $i$ th block. Here are some examples of topological operads.

Example 3.2 For a topological space $X$, the endomorphism operad $\operatorname{End}(X)$ is the symmetric operad defined by $\operatorname{End}(X)(n)=\operatorname{Map}\left(X^{n}, X\right)$, such that the composition $\circ_{i}$ is insertion in the $i$ th variable.

For $f \in \operatorname{End}(X)(k)$ and $g \in \operatorname{End}(X)(l)$

$$
f \circ_{i} g \in \operatorname{End}(X)(k+l-1)=\operatorname{Map}\left(X^{k+l-1}, X\right)
$$

is

$$
\left(f \circ \circ_{i} g\right)\left(x_{1}, \ldots, x_{k+l-1}\right)=f\left(x_{1}, \ldots, x_{i-1}, g\left(x_{i}, \ldots, x_{i+l-1}\right), x_{i+l}, \ldots, x_{k+l-1}\right) \text {. }
$$

The identity of $X$ is the unit of $\operatorname{End}(X)$.

We say that a space $A$ is acted on by a (symmetric) operad $O$, or that it is an $O$-algebra, if we are given a morphism of (symmetric) operads $O \rightarrow \operatorname{End}(A)$.

The concepts of (symmetric) operads and their algebras can be defined likewise in any (symmetric) monoidal category.

Example 3.3 Dually to Example 3.2, for a topological space $X$ the coendomorphism operad Coend $(X)$ is the symmetric operad defined by

$$
\operatorname{Coend}(X)(n)=\operatorname{Map}\left(X, X^{n}\right)=\operatorname{Map}(X, X)^{n} .
$$


Given maps $f=\left(f_{1}, \ldots, f_{k}\right): X \rightarrow X^{k}$ and $g=\left(g_{1}, \ldots, g_{l}\right): X \rightarrow X^{l}$ the composition is defined by

$$
f \circ_{i} g=\left(f_{1}, \ldots, f_{i-1}, g_{1} \circ f_{i}, \ldots, g_{l} \circ f_{i}, f_{i+1}, \ldots, f_{k}\right): X \rightarrow X^{k+l-1} .
$$

Example 3.4 Let $M$ be a topological monoid. Then there is an operad $\underline{M}$ with $\underline{M}(n)=M^{n}$. The composition is

$$
\left(x_{1}, \ldots, x_{m}\right) \circ_{i}\left(y_{1}, \ldots, y_{n}\right)=\left(x_{1}, \ldots, x_{i-1}, x_{i} y_{1}, \ldots, x_{i} y_{n}, x_{i+1}, \ldots, x_{m}\right) .
$$

Actually the coendomorphism operad of Example 3.3 is the special case with $M=$ $\operatorname{Map}(X, X)$ endowed with the multiplication $f \cdot g=g \circ f$.

Example 3.5 The associative operad Ass is the operad such that $\operatorname{Ass}(n)$ is a point for each $n$. This is a non-symmetric operad and is the final object in the category of non-symmetric topological operads. An algebra over Ass is exactly a topological monoid.

Example 3.6 The little $n$-discs operad $\mathcal{D}_{n}$ by Boardman and Vogt is the main example that motivated historically the introduction of operads [1] (May [16]). The space $\mathcal{D}_{n}(k)$ is the space of $k$-tuples of self-maps of the unit $n$-disc $f_{i}: D_{n} \rightarrow D_{n}, \quad i=1, \ldots, k$ obtained by composing translations and dilatations, ie $f_{i}(z)=\lambda_{i} z+z_{i}, \quad \lambda_{i} \in \mathbb{R}_{+}, z_{i} \in$ $\mathbb{R}^{n}$, and such that $f_{i}\left(D_{n}\right) \cap f_{j}\left(D_{n}\right)=\varnothing$ for $i \neq j$. This is a symmetric suboperad of the coendomorphism operad of the disc $D_{n}$. We can associate a picture to an element of $\mathcal{D}_{n}$ drawing the images of the maps.

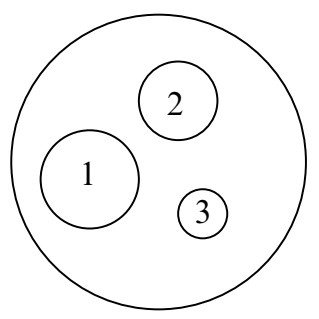

The $n$-fold loop space $\Omega^{n}(X) \cong \operatorname{Map}\left(D_{n}, S^{n-1} ; X, x_{0}\right)$ on a based space $\left(X, x_{0}\right)$ is an algebra over the symmetric operad/ $D_{n}$. The structure map is adjoint to the map

$$
\theta_{n}: D_{n}(k) \times \Omega^{n}(X)^{k} \rightarrow \Omega^{n}(X)
$$

sending $\left(f_{1}, \ldots, f_{k} ; \omega_{1}, \ldots, \omega_{k}\right)$, for $f_{i}: D_{n} \rightarrow D_{n}$ and $\omega_{j}: D_{n} \rightarrow X$, to the $n$-fold loop

$$
\theta_{n}\left(f_{1}, \ldots, f_{k} ; \omega_{1}, \ldots, \omega_{k}\right): D_{n} \rightarrow X
$$


coinciding with $\omega_{i} \circ\left(f_{i}\right)^{-1}$ on the image $f_{i}\left(D_{n}\right)$ for $i=1, \ldots, k$ and sending any point that is not in the image of any $f_{i}$ to the base point $x_{0} \in X$.

A partial converse holds, the recognition principle.

Proposition 3.7 (Boardman-Vogt [1] May [16]) Let $A$ be an algebra over $\mathcal{D}_{n}$, with $n \geq 1$. If $A$ is connected then $A$ is weakly homotopy equivalent to a $n$-fold loop space.

More generally this is true if $A$ is group-like. This means that the set of components $\pi_{0}(A)$, that is always a monoid by the action of $\mathcal{D}_{n}$, is also a group.

Example 3.8 The framed little $n$-discs operad $f \mathcal{D}_{n}$ introduced by Getzler in [6] contains $\mathcal{D}_{n}$, and is defined similarly as $\mathcal{D}_{n}$ except that the self-maps in the definition can be composed by oriented rotations.

Precisely $f \mathcal{D}_{n}(k)$ is the space of $k$-tuples of self-maps $f_{i}: D_{n} \rightarrow D_{n}, i=1, \ldots, k$ such that $f_{i}(z)=\lambda_{i} A_{i} z+z_{i}$ with $\lambda_{i} \in \mathbb{R}_{+}, A_{i} \in S O(n), z_{i} \in \mathbb{R}^{n}$, and such that $f_{i}\left(D_{n}\right) \cap f_{j}\left(D_{n}\right)=\varnothing$ for $i \neq j$. Also $f \mathcal{D}_{n}$ is a suboperad of the coendomorphism operad of the disc $D_{n}$. There is an obvious inclusion of operads $\mathcal{D}_{n} \subset f \mathcal{D}_{n}$.

The operad $f \mathcal{D}_{n}$ acts on the $n$-fold loop space $\Omega^{n}(X)$ of any space $X$ with based $S O(n)$-action.

The recognition principle for algebras over $f \mathcal{D}_{n}$ is the following.

Proposition 3.9 (Salvatore-Wahl [23]) Let $A$ be an algebra over $f \mathcal{D}_{n}$. If $A$ is group-like then it is weakly equivalent to $\Omega^{n}(X)$, where $X$ has a based $S O(n)$-action, via a zig-zag of based $S O(n)$-equivariant maps.

Definition 3.10 (The language of trees) Here we follow the terminology of the book by Markl, Shnider and Stasheff [15], with the important difference that we work with non-symmetric rather than symmetric operads, and with planar rather than non-planar trees. An important point about non-symmetric operads is that it makes sense to compose elements of the operad along a rooted planar tree. This is a planar graph with no cycles, where we allow some open half-edges. The root is one of these half-edges, and the remaining ones are the leaves. All other edges are called internal edges. There is a unique structure of directed graph on a rooted planar tree such that there is a directed path from each point to the root. Given a rooted planar tree $T$, the arity $|v|$ of a vertex $v$ is the number of incoming edges. The counterclockwise ordering induces an ordering on the incoming edges of a vertex. It is customary to draw the tree so that the root is on the bottom and the edges are directed downward. Here is an example of a tree with 4 vertices of arity $|x|=0,|y|=1,|z|=2$ and $|t|=3$. 


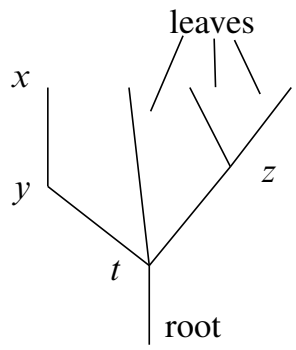

Given a topological operad $O$, let us consider the product $O(T)=\prod_{v} O(|v|)$ indexed over all vertices $v$ of $T$. This product can be thought of as the space of trees of shape $T$ with vertices labeled by the operad $O$. If a tree $T^{\prime}$ is obtained from $T$ by contracting an internal edge $e: v \rightarrow w$, and $e$ is the $i$ th incoming edge of $w$, then the composition map

$$
\circ_{i}: O(|w|) \times O(|v|) \rightarrow O(|w|+|v|-1)
$$

induces a map $O(T) \rightarrow O\left(T^{\prime}\right)$. The axioms of Definition 3.1 guarantee that for iterated contractions the result does not depend on the order. In the end there is a well defined composition map $O(T) \rightarrow O(n)$, where $n$ is the number of leaves of the tree $T$. This can be expressed by the notion of a triple. We recall that a triple $(F, \mu, u)$ in a category $\mathcal{C}$ is a monoid in the monoidal category of endofunctors of $\mathcal{C}$, ie a functor $F: \mathcal{C} \rightarrow \mathcal{C}$ together with natural transformations $\mu: F \circ F \rightarrow F$ and $u: \operatorname{id}_{\mathcal{C}} \rightarrow F$ satisfying associative and unital properties. An algebra over the triple is an object $A$ of $\mathcal{C}$ with a morphism $\rho: F(A) \rightarrow A$ compatible in an appropriate sense with $\mu$ and $u$. In our case $\mathcal{C}$ is the category of sequences of topological spaces $X=(X(n)) \quad(n \in \mathbb{N})$ with a distinguished base point $\iota \in X(1)$. The functor $F$ sends a sequence $X$ to a sequence $F(X)$, where $F(X)(n)$ is the space of rooted planar trees on $n$ leaves of all possible shapes, labelled by elements of $X$, modulo the relation deleting arity 1 vertices labeled by the base point $\iota \in X(1)$. The natural transformation $\mu: F \circ F \rightarrow F$ sends a tree $T$ with labels that are themselves labeled trees to the labeled tree obtained cutting a neighbourhood of each vertex of $T$ and gluing in its label. The unit transformation $u$ : id $\rightarrow F$ identifies a point to a corolla labeled by the same point, where a corolla is a tree with a single vertex. An algebra over this triple is exactly a non-symmetric topological operad. In other words $F(X)$ is the sequence underlying the free operad generated by $X$.

\section{Cacti operads}

We claim that the operads used by various authors dealing with the Deligne conjecture are very similar. 
We recall that two topological operads $A, B$ are said to be equivalent if there is a zig-zag of operad morphisms that are levelwise homotopy equivalences connecting $A$ and $B$. An $E_{n}$ operad is an operad equivalent to the little $n$-discs $\mathcal{D}_{n}$. Voronov introduced in [25] the cacti operad Cacti that is equivalent to the framed little 2-discs operad $f \mathcal{D}_{2}$ if we forget constants, ie the arity 0 part. A variation of the cacti operad that is instead equivalent to the little 2 -discs $\mathcal{D}_{2}$, if we forget constants, is the spineless cacti operad, introduced by Kaufmann [10].

We will describe in this section an $E_{2}$ operad constructed by McClure and Smith in [18], that we call $M S$. This operad is closely related to the spineless cacti operad.

The space $M S(n)$ splits as a product of a finite CW-complex $\mathcal{F}(n)$ and a contractible factor. We will give a geometric description of $\mathcal{F}(n)$.

Definition 4.1 An element $x \in \mathcal{F}(n)$ is a partition of the circle $S^{1}$ into $n$ closed 1 -manifolds $I_{j}(x) \quad(j=1, \ldots, n)$ with equal length (=measure), and pairwise disjoint interiors, such that there is no cyclically ordered 4-tuple $\left(z_{1}, z_{2}, z_{3}, z_{4}\right)$ in $S^{1}$ with $z_{1}, z_{3} \in \stackrel{\circ}{I}_{j}(x), z_{2}, z_{4} \in \stackrel{\circ}{I}_{k}(x)$ and $j \neq k$.

The figure shows an element of $\mathcal{F}(4)$.

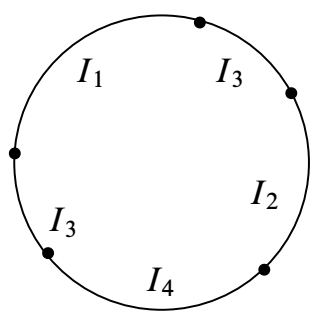

We can define on $\mathcal{F}(n)$ a topology induced by a metric such that two partitions $x, y \in \mathcal{F}(n)$ are close if the manifolds $I_{j}(x)$ and $I_{j}(y)$ are close for each $j$. For example the metric $d(x, y)=\sum_{j=1}^{n} l\left(I_{j}(x) \backslash \stackrel{\circ}{I}_{j}(y)\right)$ does the job, where $l$ denotes the measure. The symmetric group $\Sigma_{n}$ acts on $\mathcal{F}(n)$ by reindexing the labels.

Clearly $\mathcal{F}(1)$ is a point. Let $\pi: I \rightarrow I / \partial I$ be the projection. The space $\mathcal{F}(2)$ is $\Sigma_{2}-$ equivariantly homeomorphic to a circle with antipodal $\Sigma_{2}$-action. The homeomorphism $I / \partial I \cong \mathcal{F}(2)$ sends $\pi(x)$ into the pair of intervals $\left\{\pi\left[x, x+\frac{1}{2}\right], \pi\left[x+\frac{1}{2}, x+1\right]\right\}$. 
The cell structure The pullback of a partition $x \in \mathcal{F}(n)$ to $I$ via $\pi: I \rightarrow I / \partial I \cong S^{1}$ determines a partition of $I$ into say $k$ intervals labelled by natural numbers between 1 and $n$. Any component of $\pi^{-1}\left(I_{j}(x)\right)$ is labelled by $j$. This gives an ordered sequence of boundary points $0=x_{0}<x_{1}<\cdots<x_{k-1}<x_{k}=1$, together with the sequence of labels $X=\left(X_{1}, \ldots, X_{k}\right)$, such that $X_{i}$ is a label of the interval $\left[x_{i-1}, x_{i}\right]$. It follows that $\mathcal{F}(n)$ is a regular CW-complex with one cell for each fixed sequence of labels $X$. The closed cell indexed by a sequence $X$ of length $k$ corresponds to the subspace of the $(k-1)$-simplex containing the elements

$$
0=x_{0} \leq x_{1} \leq \cdots \leq x_{k-1} \leq x_{k}=1
$$

such that

$$
\sum_{i \mid X_{i}=j}\left(x_{i}-x_{i-1}\right)=1 / n
$$

for each $j=1, \ldots, n$. This subspace is homeomorphic to the product of simplices $\prod_{j=1}^{n} \Delta^{d(j)}$, where $d(j)$ is one less than the number of components (intervals) of $\pi^{-1}\left(I_{j}(x)\right)$.

The upshot is that cells of $\mathcal{F}(n)$ correspond to those finite sequences $X=\left(X_{i}\right)$ with values in $\{1, \ldots, n\}$ such that:

(1) all values between 1 and $n$ appear in $X$,

(2) two adjacent values in $X$ are distinct,

(3) the sequence $X$ does not have a subsequence of the form $\{i, j, i, j\}$ with $i \neq j$.

Given a partition $x \in \mathcal{F}(n)$ and an index $j$, let us consider the quotient space of $S^{1}$ under the relation that identifies points in the same component (closed interval) of $\overline{S^{1} \backslash I_{j}(x)}$. The result is a circle with a base point, the image of the base point of $S^{1}$. If we rescale this circle by the factor $n$ and identify it to $S^{1}$, then we see the quotient map as a based self-map $\pi_{j}: S^{1} \rightarrow S^{1}$.

We say that $c(x):=\left(\pi_{1}, \ldots, \pi_{n}\right): S^{1} \rightarrow\left(S^{1}\right)^{n}$ is the cactus map and its image is the cactus $C_{x}$ associated to $x$.

The map $c: \mathcal{F}(n) \rightarrow \operatorname{Map}\left(S^{1},\left(S^{1}\right)^{n}\right)$ is an embedding, but the image does not form a suboperad of the coendomorphism operad Coend $\left(S^{1}\right)$ of Example 3.3.

The cactus $C_{x}$ is the union of $n$ circles, the lobes $S_{j}(x)(j=1, \ldots, n)$, that are the images under $c(x)$ of the submanifolds $I_{j}(x)$. The base point $P \in C_{x}$ is the image of the base point $1 \in S^{1}$ under the cactus map $c(x): S^{1} \rightarrow C_{x}$.

Here is an example of a cactus with the indexes of the lobes. 


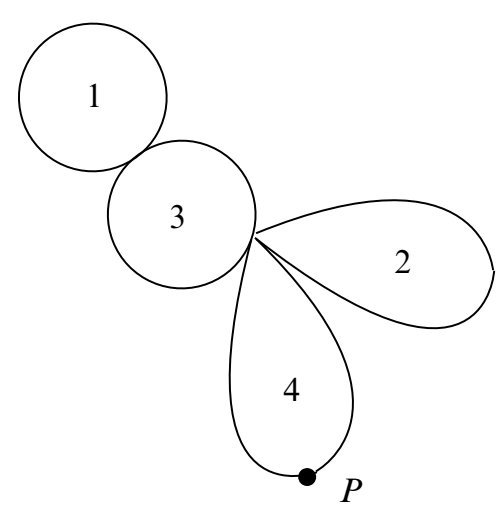

Definition 4.2 (The associated tree) One can associate to each partition $x \in \mathcal{F}(n)$ a rooted planar tree (constant on open cells) constructed as follows. The tree is the graph that has a vertex for each lobe (ie for each number between 1 and $n$ ), a vertex for each intersection point of distinct lobes, and an edge between the vertex of an intersection point and the vertex of each lobe it belongs to. Let $P \in C_{x}$ be the base point. If $P$ is an intersection point then the root vertex is its vertex. Otherwise $P$ belongs to a unique lobe and the root vertex is the vertex of that lobe. The root is an additional half-open edge incident to the root vertex. The tree is a ribbon graph with a cyclic ordering of the edges incident to a given vertex. The ordering is induced by the standard orientation of the circle via the map $c(x): S^{1} \rightarrow C_{x}$. The tree admits a planar embedding such that the cyclic ordering is compatible with the standard orientation of the plane (Voronov [25]). It is possible to embed the cactus $C_{x}$ into the plane, in a way that this is compatible with the embedding of the tree on the intersection points, as shown in the next figure. The tree is the union of corollas inscribed in the lobes.

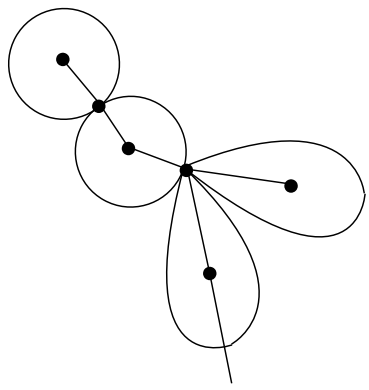

More operads McClure and Smith constructed in [17] first an operad $\mathcal{C}^{\prime}$ that is equivalent to little $2-$ discs $\mathcal{D}_{2}$ away from the arity 0 part, because $\mathcal{C}^{\prime}(0)$ is empty but $\mathcal{D}_{2}(0)$ is a point. As a $\Sigma_{n}$-space $\mathcal{C}^{\prime}(n)=\mathcal{F}(n) \times \stackrel{\circ}{\Delta}_{n-1}$. 
The space $\mathcal{C}^{\prime}(n)$ is naturally homeomorphic to a space of partitions defined similarly as in Definition 4.1, except that one drops the condition that all manifold must have the same length. In this setting there is also a cactus map $\mathcal{C}^{\prime} \rightarrow \operatorname{Coend}\left(S^{1}\right)$ that is an embedding onto a symmetric suboperad of $\operatorname{Coend}\left(S^{1}\right)$ and defines an operad structure on $\mathcal{C}^{\prime}$.

The spineless cacti operad Cact by Kaufmann [10] is actually a pseudo-operad, in the sense that is associative, non-unital, and is isomorphic to $\mathcal{C}^{\prime} \times R$, where $R$ is the constant pseudo-operad $R(n)=\mathbb{R}_{+}$with composition $\mathbb{R}_{+} \times \mathbb{R}_{+} \rightarrow \mathbb{R}_{+}$equal to the projection onto the first factor. The extra parameter corresponds to the length of the parametrizing circle in [10].

McClure and Smith extended in [17] the operad $\mathcal{C}^{\prime}$, that has $\mathcal{C}^{\prime}(0)$ empty, to an operad $\mathcal{C}$ equivalent to the little 2 -discs operad such that $\mathcal{C}(0)$ is a base point. The operad $\mathcal{C}$ is the smallest suboperad of $\operatorname{Coend}\left(S^{1}\right)$ containing $\mathcal{C}^{\prime}$ and the base point in arity 0.

Later on McClure and Smith defined a larger operad that is denoted $\mathcal{D}_{2}$ in [18]. We present a geometric construction of such operad.

Definition 4.3 Let $\operatorname{Mon}(I, \partial I)$ be the space of self-maps of the circle $S^{1} \cong I / \partial I$ induced by weakly monotone self-maps of the interval $I$ restricting to the identity on the boundary $\partial I$. We define $M S(n)=\mathcal{F}(n) \times \operatorname{Mon}(I, \partial I)$ for $n>0$. We also define $M S(0)$ to be a point.

Remark 4.4 The space $\operatorname{Mon}(I, \partial I)$ is homeomorphic to the contractible space $\operatorname{Tot}\left(\Delta^{*}\right)$, ie the monoid of cosimplicial endomorphisms of the cosimplicial space $\Delta^{*}$. The homeomorphism $T: \operatorname{Mon}(I, \partial I) \rightarrow \operatorname{Tot}\left(\Delta^{*}\right)$ sends $f: I \rightarrow I$ to the sequence of maps $T(f)^{k}: \Delta^{k} \rightarrow \Delta^{k}$ with

$$
T(f)^{k}:\left(0 \leq x_{1} \leq \cdots \leq x_{k} \leq 1\right) \mapsto\left(0 \leq f\left(x_{1}\right) \leq \cdots \leq f\left(x_{k}\right) \leq 1\right) .
$$

There is an embedding $\zeta_{n}: M S(n) \rightarrow \operatorname{Coend}\left(S^{1}\right)(n)$ sending, for $n>0, x \in \mathcal{F}(n)$ and $f \in \operatorname{Mon}(I, \partial I)$ to the composition $c(x) \circ f: S^{1} \rightarrow\left(S^{1}\right)^{n}$. The following characterization is immediate.

Proposition 4.5 The image $\zeta_{n}(M S(n))$ is the space of based maps $g: S^{1} \rightarrow\left(S^{1}\right)^{n}$ such that

(1) $\pi_{i} \circ g$ is a weakly monotone degree 1 map for each $i=1, \ldots, n$,

(2) there is a partition of $S^{1}$ into closed intervals intersecting at the boundary such that on each interval $\pi_{i} \circ g$ is constant for all indexes $i$ except one special index, 
(3) the counterclockwise sequence of special indices does not contain a subsequence of the form $\{i, j, i, j\}$ with $i \neq j$.

It follows that the image of the embedding $\zeta$ is a symmetric suboperad of $\operatorname{Coend}\left(S^{1}\right)$. This gives an operad structure on $M S$.

Proposition 4.6 The operad $M S$ is isomorphic to the $E_{2}$ operad denoted $\mathcal{D}_{2}$ in [18].

A proof will be presented in the appendix.

We build next an operad $f M S$ that is equivalent to the framed little 2-discs. It is related to $M S$ in the same way as Voronov's cacti operad is related to Kaufmann's spineless cacti operad.

Definition 4.7 Let us consider the $\Sigma_{n}$-space $f M S(n)=M S(n) \times\left(S^{1}\right)^{n}$ for any natural number $n$. There is an embedding $c^{\prime}: f M S(n) \rightarrow \operatorname{Coend}\left(S^{1}\right)(n)$ sending

$$
\left(x, f,\left(z_{1}, \ldots, z_{n}\right)\right) \mapsto\left(L_{z_{1}} \times \cdots \times L_{z_{n}}\right) \circ c(x) \circ f
$$

where $L_{z}: S^{1} \rightarrow S^{1}$ is multiplication by $z \in S^{1}$. A map $g: S^{1} \rightarrow\left(S^{1}\right)^{n}$ belongs to the image $c^{\prime}(f M S(n))$ if and only if it is an unbased map satisfying the conditions $1,2,3$ of Proposition 4.5. It follows that the image of $c^{\prime}$ is a symmetric suboperad of Coend $\left(S^{1}\right)$, and this defines an operad structure on $f M S$.

In order to prove that $f M S$ is equivalent to $f \mathcal{D}_{2}$ we need a lemma. The lemma is exposed as [3, Theorem 2.2.2] and follows from [23, Theorem 7.3], that is a generalization of a similar result by Fiedorowicz for the little 2-discs operad.

Lemma 4.8 Let $O$ be a symmetric operad together with a $\Sigma_{k}$-equivariant homotopy equivalence $O(k) \simeq f \mathcal{D}_{2}(k)$ for each $k$, and together with an operad map $E \rightarrow O(k)$ from an $E_{1}$-operad $E$. Then $O$ is equivalent to $f \mathcal{D}_{2}$.

Proposition 4.9 The operad $f M S$ is equivalent to the framed little 2-discs operad $f \mathcal{D}_{2}$.

Proof We know from Proposition 4.6 that $M S$ is an $E_{2}$-operad, and this gives for each $k$ a $\Sigma_{k}$-equivariant homotopy equivalence

$$
f M S(k)=M S(k) \times\left(S^{1}\right)^{k} \simeq \mathcal{D}_{2}(k) \times\left(S^{1}\right)^{k}=f \mathcal{D}_{2}(k) .
$$

In addition $M S \subset f M S$ contains an $E_{1}$-operad $M S_{1}$ defined in the appendix. By Lemma 4.8 this concludes the proof. 
For $y=\left(x, f,\left(z_{1}, \ldots, z_{n}\right)\right) \in f M S(n)$ we denote by $C_{y}$ and call cactus the image of $\left(L_{z_{1}} \times \cdots \times L_{z_{n}}\right) \circ c(x): S^{1} \rightarrow\left(S^{1}\right)^{n}$. We also call lobe and denote $S_{j}(y)$ the image of $I_{j}(x)$ via the same map. The lobe has a standard identification to $S^{1}$ via the projection $\left(S^{1}\right)^{n} \rightarrow S^{1}$ onto the $j$ th coordinate. The base point of the cactus is the image of the base point of $S^{1}$. In general the base points of the lobes are not intersection points or the base point of the cactus as in the spineless case.

The connection to Voronov's cacti operad Cacti, that actually is a pseudo-operad, is as follows. The operad $f M S$ contains an operad $f \mathcal{C}^{\prime}$ with $f \mathcal{C}^{\prime}(n)=\mathcal{C}^{\prime}(n) \times\left(S^{1}\right)^{n}$. In particular $f \mathcal{C}^{\prime}(0)$ is empty. The inclusion $f \mathcal{C}^{\prime}(n) \rightarrow f M S(n)$ is a $\Sigma_{n}$-equivariant homotopy equivalence for all $n>0$, and there is an isomorphism of pseudo-operads Cacti $\cong f \mathcal{C}^{\prime} \times R$.

\section{The non-cyclic Deligne conjecture}

We shall reprove in this section the result by McClure and Smith. We need the following definition.

Definition 5.1 A topological operad with multiplication $O$ is a non-symmetric operad $O$ together with a morphism Ass $\rightarrow O$ from the associative operad.

Equivalently $O$ is a non-symmetric operad in the monoidal category of based spaces with the cartesian product as tensor product. Also equivalently $O$ is a topological operad with points $m \in O(2)$ (the multiplication) and $u \in O(0)$ (the unit of the multiplication) such that

$$
\begin{aligned}
m \circ_{1} m & =m \circ_{2} m \in O(3) \\
m \circ_{1} u & =m \circ_{2} u=\iota \in O(1) .
\end{aligned}
$$

Remark 5.2 There is a triple $F_{*}$ in the category of sequences of based spaces such that an operad with multiplication is exactly an algebra over $F_{*}$. The triple $F_{*}$ is closely related to the triple $F$ of Definition 3.10. Namely, for a sequence $X, F_{*}(X)$ is the quotient of $F(X)$ under the identification relation induced by collapsing internal edges with both vertices labeled by base points.

We can relate now cosimplicial spaces and operads. 
Definition 5.3 An operad $O$ with multiplication defines a cosimplicial space $O^{*}$ sending $[k]$ to $O(k)$. The coface operator $d^{i}: O(k) \rightarrow O(k+1)$ is defined by

$$
\begin{aligned}
d^{i}(x) & =x \circ_{i} m \text { for } 1 \leq i \leq k \\
d^{0}(x) & =m \circ_{2} x \\
d^{k+1}(x) & =m \circ_{1} x .
\end{aligned}
$$

The codegeneracies $s^{i}: O(k) \rightarrow O(k-1)$ are defined by

$$
s^{i}(x)=x \circ_{i+1} u \text {. }
$$

Theorem 5.4 (McClure-Smith [18]) Let $O$ be an operad with multiplication. Then the totalization $\operatorname{Tot}\left(O^{*}\right)$ admits the action of the $E_{2}$ operad $M S$.

\section{Proof}

We explain geometrically the action of $M S$ on $\operatorname{Tot}\left(O^{*}\right)$. We need to describe the action of $M S$ on $\operatorname{Tot}\left(O^{*}\right)$ via maps

$$
\theta_{m}: M S(m) \times \operatorname{Tot}\left(O^{*}\right)^{m} \rightarrow \operatorname{Tot}\left(O^{*}\right) .
$$

Given $(x, f) \in M S(m)=\mathcal{F}(m) \times \operatorname{Tot}\left(\Delta^{*}\right)$ and $m$ cosimplicial maps

$$
a_{1}^{*}, \ldots, a_{m}^{*} \in \operatorname{Tot}\left(O^{*}\right)
$$

we want to define a cosimplicial map

$$
a^{*}=\theta_{m}\left(x, f ; a_{1}^{*}, \ldots, a_{m}^{*}\right) \in \operatorname{Tot}\left(O^{*}\right),
$$

so we need compatible maps $a^{k}: \Delta^{k} \rightarrow O(k)$ for every non-negative integer $k$. A point of the simplex $\Delta^{k}$ is a sequence of points of the unit interval

$$
0=x_{0} \leq x_{1} \leq \cdots \leq x_{k} \leq 1 .
$$

The cactus map induces a curve

$$
\gamma=c(x) \circ f \circ \pi: I \rightarrow C_{x} \subset\left(S^{1}\right)^{m} .
$$

We construct a rooted planar tree $T$ associated to these data. We start with the tree associated to $x \in \mathcal{F}(m)$ in Definition 4.2 with its root removed. This tree has

- a vertex for each lobe,

- a vertex for each intersection point,

- edges between vertices of intersection points and vertices of their lobes. 
We call the points of the form $\gamma\left(x_{j}\right) \quad(0 \leq j \leq k)$ special points. For each special point that is not an intersection point we add a new vertex, and an edge connecting it to the vertex of the lobe containing $\gamma\left(x_{j}\right)$.

Finally for each point of the form $x_{j} \quad(0 \leq j \leq k)$ we add a leaf (half-open edge) incident to the vertex of $\gamma\left(x_{j}\right)$. The edge incident to the vertex of the base point $\gamma\left(x_{0}\right)$ is the root. The difference with the tree in Definition 4.2 is that we subdivide the former root inserting a vertex.

The tree $T$ is a planar rooted tree: the ordering of the edges incident to a vertex is induced by the standard orientation of the interval via $\gamma$ and by the ordering of the indices. Both the tree $T$ and the cactus $C_{x}$ can be embedded together consistently into the plane, so that the embeddings are compatible on points of the form $\gamma\left(x_{j}\right)$ and on intersection points. An example with $k=6$ and $m=4$ is given in the next figure.
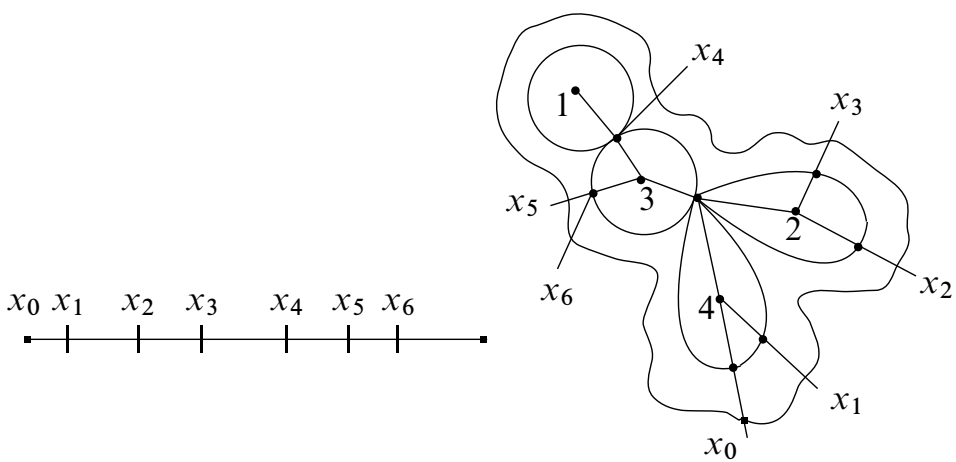

Next we label each vertex $v$ of $T$ by an element of the operad of the same arity.

If $v$ corresponds to an intersection point of lobes, or to a point of the form $\gamma\left(x_{j}\right)$, then we label it by the base point of $O(|v|)$. Let Int $\subset C_{x}$ be the set of all intersection points.

If $v$ corresponds to a lobe $S_{j}$, then let us take the set

$$
H_{j}=\left(\operatorname{Int} \cup \gamma\left(\left\{x_{1}, \ldots, x_{k}\right\}\right)\right) \cap\left(S_{j}-*\right)
$$

of special points and intersection points in the lobe that are not its base point.

Suppose that $H_{j}$ has $l_{j}$ points. The arity of $v$ is exactly $|v|=l_{j}$. There is a standard identification of the lobe $S_{j}$ with $S^{1}$ via inclusion $S_{j}(x) \subset C_{x} \subset\left(S^{1}\right)^{m}$ followed by projection onto the $j$ th factor. Pulling back $H_{j}$ via $I \rightarrow I / \partial I \cong S^{1} \cong S_{j}$ gives $l_{j}$ points in the unit interval $I$, ie a point $y_{j}$ of the simplex $\Delta^{l_{j}}$. Actually $y_{i}$ is 
in the interior of the simplex. The cosimplicial map $a_{j} \in \operatorname{Tot}\left(O^{*}\right)$ in degree $l_{j}$ $a_{j}^{l_{j}}: \Delta^{l_{j}} \rightarrow O\left(l_{j}\right)$ evaluated on $y_{j}$ gives an element of the operad $a_{j}^{l_{j}}\left(y_{j}\right) \in O\left(l_{j}\right)$ that we assign as label of $v$. In the terminology of Definition 3.10 our labelled tree is some $\Gamma \in O(T)$. The generalised composition $O(T) \rightarrow O(k)$ applied to the labelled tree $\Gamma$ defines the desired element $a^{k}\left(x_{1}, \ldots, x_{k}\right) \in O(k)$.

The resulting map $a^{*}: \Delta^{*} \rightarrow O^{*}$ is a cosimplicial map essentially because $a_{1}^{*}, \ldots, a_{m}^{*}$ are all cosimplicial maps. The maps $\theta_{m}$ define a $M S$-algebra structure on $\operatorname{Tot}\left(O^{*}\right)$. To see this we need to look at the labelled trees whose composition defines the structure maps, and use the fact that the ordering of composition by contracting edges is not relevant. More precisely we are using the fact that $O$ is an algebra over the triple $F_{*}$ of Remark 5.2.

Example 5.5 Suppose that $x \in M S(2)$, so that $C_{x}$ has two lobes, that the base point is in $S_{1}$, and that $k=1$. We wish to compute the value of the map $a^{1}: \Delta^{1} \rightarrow O(1)$ on $x_{1} \in I$. We suppose that the intersection point $Q=S_{1} \cap S_{2}$ coincides with the special point $Q=\gamma\left(x_{1}\right)$. We also assume that the curve $\gamma$ at $x_{1}$ goes from the lobe $S_{1}$ into $S_{2}$, ie $\gamma\left(\left[0, x_{1}\right]\right) \subset S_{1}$. Then $l_{1}=1, l_{2}=0$ and $y_{1}$ is the point mapping to $Q$ via $I \rightarrow S^{1} \cong S_{1}$. It follows that

$$
a^{1}\left(x_{1}\right)=\left(a_{1}^{1}\left(y_{1}\right) \circ_{1} m_{2}\right) \circ_{2} a_{2}^{0}(*) .
$$

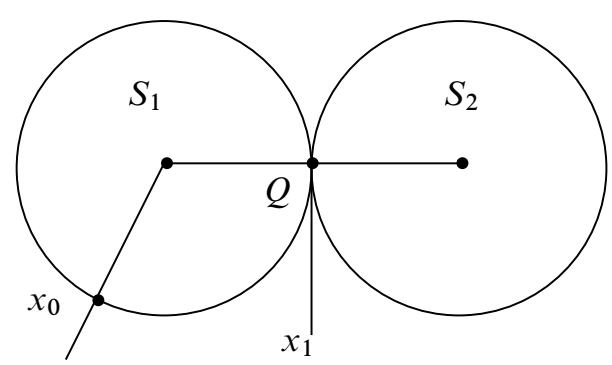

Theorem 5.4 and the recognition principle (Proposition 3.7) immediately imply the following corollary.

Corollary 5.6 If $O$ is an operad with multiplication and $\operatorname{Tot}\left(O^{*}\right)$ is group-like then $\operatorname{Tot}\left(O^{*}\right)$ has the weak homotopy type of a double loop space.

Example 5.7 Let $M$ be a topological monoid. The operad $\underline{M}$ of Example 3.4 has a multiplication by choosing $m_{2}=(1,1) \in M^{2}$, and $m_{0}=* \in M^{0}$. Theorem 5.4 tells us that the operad $M S$ acts on $\operatorname{Tot}(\omega M) \cong \Omega M \simeq \Omega^{2}(B M)$. This is consistent with Corollary 5.6. 
Example 5.8 (Knots from operads) We recall that a long knot is an embedding $\mathbb{R} \rightarrow \mathbb{R}^{n}$ that is fixed near infinity, and a framed long knot is a long knot with a trivialization of its tubular neighbourhood that is fixed near infinity. The Kontsevich operad $\mathcal{K}_{n}$ is an operad with multiplication that is equivalent to the little discs $\mathcal{D}_{n}$, which instead does not admit a multiplication. Sinha proved in [24], using Goodwillie calculus, that the homotopy totalization $\widetilde{\operatorname{Tot}}\left(\mathcal{K}_{n}^{*}\right)$ is weakly equivalent to $\operatorname{Emb}_{n} \times \Omega^{2} S^{n-1}$ for $n>3$, where $\mathrm{Emb}_{n}$ is the space of long knots in $\mathbb{R}^{n}$. The homotopy totalization of a cosimplicial space is weakly equivalent to the homotopy limit of the cosimplicial space as a diagram. One cannot apply directly Theorem 5.4 to the homotopy totalization, but there is a derived version of Theorem 5.4 by McClure and Smith ([19, Theorem 15.3]) constructing an $E_{2}$ operad that acts on the homotopy totalization of any operad with multiplication. By Corollary 5.6 the space $\mathrm{Emb}_{n} \times \Omega^{2} S^{n-1}$, which is connected by Whitney's theorem, is a double loop space for $n>3$. We proved in [22] that $\mathrm{Emb}_{n}$ itself is a double loop space for $n>3$. We also proved that the space of framed long knots $f \mathrm{Emb}_{n}$ is a double loop space, because it is weakly equivalent to $\widetilde{\operatorname{Tot}}\left(f \mathcal{K}_{n}^{*}\right)$, where $f \mathcal{K}_{n}$ is the framed Kontsevich operad, that is equivalent to the framed little $n$-discs $f \mathcal{D}_{n}$.

\section{The cyclic category}

The cyclic category $\Lambda$ was introduced by Connes, and is an extension of the category $\Delta$ by adding for each $n$ an automorphism $\tau_{n}:[n] \rightarrow[n]$ of order $n+1$, such that

$$
\begin{array}{cc}
\tau_{n} \delta_{i}=\delta_{i-1} \tau_{n-1} & 1 \leq i \leq n \\
\tau_{n} \sigma_{i}=\sigma_{i-1} \tau_{n+1} & 1 \leq i \leq n .
\end{array}
$$

As a consequence of the axioms $\tau_{n} \delta_{0}=\delta_{n}$ and $\tau_{n} \sigma_{0}=\sigma_{n}\left(\tau_{n+1}\right)^{2}$. We refer to Drinfeld [4] for a discussion of the cyclic category.

A cocyclic space is a covariant functor from $\Lambda$ to the category of topological spaces. In particular by restriction to $\Delta \subset \Lambda$ a cocyclic space has an underlying cosimplicial space, that we denote with the same name. We denote by $t_{n}$ the image of $\tau_{n}$ under the functor defining a cocyclic space.

Proposition 6.1 (Jones [9]) Let $C$ be a cocyclic space. Then the space $\operatorname{Tot}(C)$ has a natural circle action.

Example 6.2 ([9]) The cosimplicial model of the free loop space $l X$ of Example 2.5 has a compatible cocyclic space structure defined by maps $t_{n}: X^{n+1} \rightarrow X^{n+1}$ with

$$
t_{n}\left(x_{0}, \ldots, x_{n}\right)=\left(x_{1}, \ldots, x_{n}, x_{0}\right) .
$$


The resulting circle action on $\operatorname{Tot}(l X)$, modulo the identification $\operatorname{Tot}(l X) \cong L X$, coincides with the reparameterization action on the free loop space $S^{1} \times L X \rightarrow L X$ defined by $(z, \gamma) \mapsto \gamma \circ L_{z}$, where $L_{z}: S^{1} \rightarrow S^{1}$ is multiplication by $z$.

Example 6.3 Let $G$ be a topological group. The cosimplicial space $\omega G$ of Example 2.4 has a compatible cocyclic space structure defined by maps $t_{n}: G^{n} \rightarrow G^{n}$ with

$$
t_{n}\left(g_{1}, \ldots, g_{n}\right)=\left(g_{1}^{-1} g_{2}, \ldots, g_{1}^{-1} g_{n}, g_{1}^{-1}\right) \text {. }
$$

The homeomorphism $\Omega G \cong \operatorname{Tot}(\omega G)$ is $S^{1}$-equivariant, where the circle action $\theta: S^{1} \times \Omega G \rightarrow \Omega G$ on $\Omega G=\operatorname{Map}_{*}\left(S^{1}, G\right)$

$$
\theta(z, \gamma)(w)=\gamma(z)^{-1} \gamma(z w)
$$

is given by normalized reparameterization.

Example 6.4 The cocyclic space $\Lambda^{*}$ is defined so that $\Lambda^{n} \subset\left(S^{1}\right)^{n}$ is the subspace of those $(n+1)$-tuples $\left(x_{0}, \ldots, x_{n}\right)$ that are cyclically ordered with respect to the counterclockwise cyclic ordering.

Similarly as for $\Delta^{*}$ (Example 2.2) the $i$ th coface doubles the coordinate $x_{i}$, the $i$ th codegeneracy removes the coordinate $x_{i+1}$ and the automorphism $t_{n}$ acts cyclically on the coordinates by

$$
t_{n}\left(x_{0}, \ldots, x_{n}\right)=\left(x_{1}, \ldots, x_{n}, x_{0}\right) .
$$

There is a homeomorphism $\Delta^{n} \times S^{1} \cong \Lambda^{n}$ sending

$$
\left(\left(x_{1}, \ldots, x_{n}\right), z\right) \mapsto\left(z, z+x_{1}, \ldots, z+x_{n}\right)
$$

for $z \in \mathbb{R} / \mathbb{Z} \cong[0,1] /\{0,1\} \cong S^{1}$. The inclusion $\Delta^{n} \times\{1\} \rightarrow \Lambda^{n}$ makes $\Delta^{*}$ into a cosimplicial subspace of $\Lambda^{*}$.

The totalization $\operatorname{Tot}\left(X^{*}\right)$ of a cocyclic space $X^{*}$ is naturally isomorphic to the space $\operatorname{Mor}\left(\Lambda^{*}, X^{*}\right)$ of natural transformations $\Lambda^{*} \rightarrow X^{*}$ of cocyclic spaces. In particular for any space $Y$ there is a homeomorphism $L Y \cong \operatorname{Mor}\left(\Lambda^{*} \rightarrow l Y\right)$ sending a loop $\gamma$ to the sequence of maps $\left(x_{0}, \ldots, x_{k}\right) \mapsto\left(\gamma\left(x_{0}\right), \ldots, \gamma\left(x_{k}\right)\right)$. Jones' circle action on $\operatorname{Tot}\left(X^{*}\right)$ of Proposition 6.1 is induced by the circle action on $\Lambda^{*} \cong S^{1} \times \Delta^{*}$.

\section{Cyclic operads and the Deligne conjecture}

Roughly speaking a cyclic operad exchanges the roles of inputs and outputs. We give the definition by default in the non-symmetric sense. 
Definition 7.1 A cyclic operad $O$ is an operad such that $O(n)$ has an action of the cyclic group $\mathbb{Z}_{n+1}$ for each $n$, with the generator acting by $t_{n}: O(n) \rightarrow O(n)$, such that for $f \in O(m), g \in O(n)$

$$
\begin{aligned}
t_{m+n-1}\left(f \circ_{1} g\right) & =t_{n}(g) \circ_{n} t_{m}(f) \\
t_{m+n-1}\left(f \circ_{i} g\right) & =t_{m}(f) \circ_{i-1} g \quad 1<1 \leq m \\
t_{1}(\iota) & =\iota .
\end{aligned}
$$

A symmetric cyclic operad is a cyclic operad such that $O(n)$ admits the action of the symmetric group $\Sigma_{n+1}$, extending the action of $\mathbb{Z}_{n+1}$ and of $\Sigma_{n}$.

The simplest example of a cyclic operad is the associative operad Ass with trivial action of the cyclic groups.

Example 7.2 A non trivial example of non-symmetric cyclic operad is the $A_{\infty}$ operad by Stasheff [15]. In particular $\mathbb{Z}_{5}$ acts on the pentagon $A_{\infty}(4)$ by rotations.

Example 7.3 An example of symmetric cyclic operad is the operad $\mathcal{M}$ of moduli space of Riemann surfaces with boundary. Here $\mathcal{M}(n)$ is the moduli space of the surfaces with $n+1$ boundary components. The operadic composition goes by gluing surfaces at the boundary components, and $\Sigma_{n+1}$ acts on the labels of the components. The genus zero part $\mathcal{M}_{0}$ is a cyclic operad equivalent to the framed little discs operad $f \mathcal{D}_{2}$.

Example 7.4 Budney defines in [2] the operad of conformal balls $\mathcal{C B}_{n}$ that is symmetric cyclic, and equivalent to the framed little $n$-discs $f \mathcal{D}_{n}$.

Definition 7.5 (The triple for cyclic operads) Getzler and Kapranov described in [7] the triple for symmetric cyclic operads. We describe the analog triple in the nonsymmetric case. A cyclic sequence is a sequence of spaces $X(n), n \in \mathbb{N}$ together with an action of $\mathbb{Z}_{n+1}$ on $X(n)$ for each $n$. We also assume that $X(1)$ has a base point in $X(1)$ fixed by the $\mathbb{Z}_{2}$-action. There is a triple $F^{+}$on the category of cyclic sequences. Given a cyclic sequence $X$, if we forget the actions of the cyclic groups then $F^{+}(X)$ agrees with $F(X)$. By definition $F(X)(n)$ is the space of rooted labelled trees with $n$ leaves, or equivalently with $n+1$ half edges. The group action of $\mathbb{Z}_{n+1}$ on $F(X)(n)$ switches the roots. The action of $[m] \in \mathbb{Z}_{n+1}$ on some $T \in F(X)(n)$ sets the new root as the $m$ th half-edge in the counterclockwise cyclic ordering after the old root. The new root defines a new structure of directed graph on the labelled tree $T$. For each vertex $v$ of arity $p$, or equivalently of valence $p+1$, there is a unique outgoing edge $v_{o}$ (respectively $v_{n}$ ) in the old (respectively new) directed graph structure. Suppose 
that $v_{n}$ is the $q$ th edge after $v_{o}$ in the counterclockwise ordering of the edges incident to $v$. The new label of $v$ is the result of the action of $[q] \in \mathbb{Z}_{p+1}$ on the old label in $X(p)$. Given a cyclic operad $O^{\prime}$, by virtue of Definition 7.1, the action of the triple $F\left(O^{\prime}\right) \rightarrow O^{\prime}$ respects the action of the cyclic groups. This shows that $O^{\prime}$ is an algebra over the triple $F^{+}$. Vice versa an algebra $O$ over the triple $F^{+}$is a cyclic operad. The Definition 7.1 is satisfied by restricting the action $F(O) \rightarrow O$ to labelled trees with one internal edge.

Definition 7.6 A cyclic operad with multiplication is an operad $O$ together with a morphism of cyclic operads Ass $\rightarrow O$.

Equivalently $O$ is an operad with multiplication $m_{2} \in O(2)$, and a cyclic operad, such that $t_{2}\left(m_{2}\right)=m_{2}$.

There is a triple $F_{*}^{+}$describing cyclic operads with multiplication. The triple $F_{*}^{+}$is defined on the category of cyclic sequences of based spaces, with the base points fixed by the cyclic group action. If we ignore the cyclic group action, then $F_{*}^{+}$agrees with the triple $F_{*}$ of Remark 5.2. The action of the cyclic groups is defined similarly as for $F^{+}$in Definition 7.5 .

We are now ready to state the main theorem.

Theorem 7.7 Let $O$ be a cyclic operad with multiplication. Then the operad fMS acts on $\operatorname{Tot}\left(O^{*}\right)$.

Proof We need to describe the action of $f M S$ on $\operatorname{Tot}\left(O^{*}\right)$ via maps

$$
\theta_{m}: f M S(m) \times \operatorname{Tot}\left(O^{*}\right)^{m} \rightarrow \operatorname{Tot}\left(O^{*}\right) .
$$

Given $x \in f M S(m)$, and cocyclic maps

$$
a_{1}^{*}, \ldots, a_{m}^{*} \in \operatorname{Tot}\left(O^{*}\right)=\operatorname{Mor}\left(\Lambda^{*}, O^{*}\right),
$$

we want to define a cocyclic map

$$
a^{*}:=\theta_{m}\left(x ; a_{1}^{*}, \ldots, a_{m}^{*}\right) \in \operatorname{Tot}\left(O^{*}\right)=\operatorname{Mor}\left(\Lambda^{*}, O^{*}\right)
$$

so we need compatible maps $a^{k}: \Lambda^{k} \rightarrow O(k)$ for $k \in \mathbb{N}$.

The construction is similar to the one for non-cyclic operads in the proof of Theorem 5.4 .

Let $\gamma=c^{\prime}(x): S^{1} \rightarrow\left(S^{1}\right)^{m}$ be the parametrization of the cactus $C_{x}$ associated to $x$ in Definition 4.7. Given a system of cyclically ordered points $\left(x_{0}, \ldots, x_{k}\right) \in \Lambda^{k}$ on the 
circle $S^{1}$ we obtain via $\gamma: S^{1} \rightarrow C_{x}$ a system of special points $\left(\gamma\left(x_{0}\right), \ldots, \gamma\left(x_{k}\right)\right)$ on the cactus $C_{x}$. We build a rooted labelled tree $\Gamma$ almost exactly as in the proof of Theorem 5.4. There is only a slight difference in the definition of the label of a vertex associated to a lobe. Let $S_{j}$ be a lobe of $C_{x}$ and let $H_{j} \subset S_{j}$ be the finite subset of all intersection points of $S_{j}$ with other lobes and of all special points of the form $\gamma\left(x_{i}\right)$ in the lobe. We have a preferred based identification of $S_{j}$ with $S^{1}$, but in general the base point is not an intersection point or $\gamma\left(x_{0}\right)$ as in the non-cyclic case. In the next figure we give an example for $m=3$ and $k=3$. The base point of the cactus is $P$ and the base points of the lobes are $P_{1}, P_{2}, P_{3}$. Only the vertices of the lobes are indicated.

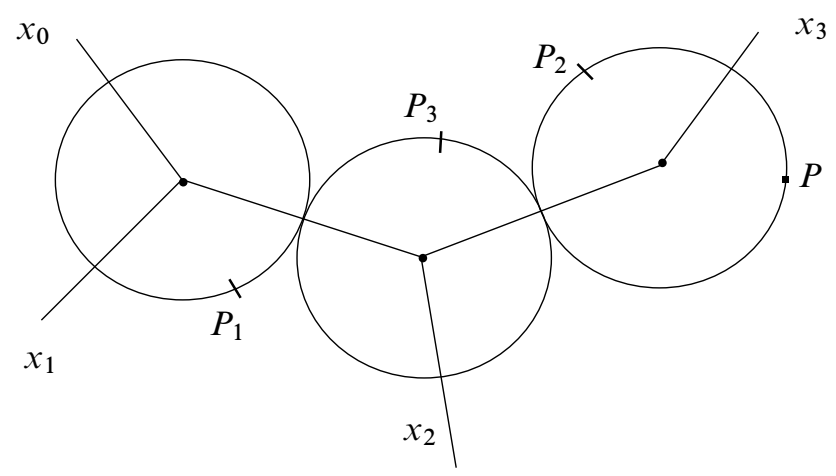

Under the identification $S^{1} \cong S_{j}$, the subset $H_{j} \subset S_{j}$ corresponds to a cyclically ordered sequence of points on $S^{1}$, that is an element $y_{j} \in \Lambda^{l_{j}}=\Delta^{l_{j}} \times S^{1}$ (actually $y_{j}$ is in the interior), where $l_{j}$ is one less than the cardinality of $H_{j}$. The map $a_{j}^{l_{j}}: \Lambda^{l_{j}} \rightarrow O\left(l_{j}\right)$ defines the label $a_{j}^{l_{j}}\left(y_{j}\right) \in O\left(l_{j}\right)$ of the vertex of $S_{j}$. We define $a^{k}\left(x_{0}, \ldots, x_{k}\right) \in O(k)$ as the composition of all labels of the rooted labelled tree $\Gamma$. The crucial point is that the map $a^{k}: \Lambda^{k} \rightarrow O(k)$ is equivariant with respect to the action of $\mathbb{Z}_{k+1}$, because $O$ is a cyclic operad. Moreover the map $a^{*}: \Lambda^{*} \rightarrow O^{*}$ respects cofaces and codegeneracies because each $a_{i}^{*}$ does so. Finally the structure maps $\theta_{*}$ make $\operatorname{Tot}\left(O^{*}\right)$ into a $f M S$-algebra, since $O$ is an algebra over the triple $F_{*}^{+}$.

Example 7.8 For a group $G$ the operad $\underline{G}$ is a cyclic operad with multiplication. The operad with multiplication is in Example 3.4 with $M=G$, and the cyclic group action in Example 6.2. These are compatible because

$$
t_{2}\left(m_{2}\right)=t_{2}(1,1)=(1,1)=m_{2} .
$$




\section{By Theorem 7.7}

$$
\operatorname{Tot}\left(\underline{G}^{*}\right) \cong \Omega G \simeq \Omega^{2} B G
$$

has the action of an operad equivalent to the little framed 2-discs. By Proposition 3.9 $B G$ is equivalent to a based space with a based $S^{1}$-action. We expect the action to be trivial, up to a zig-zag of based equivariant maps that are non-equivariant weak equivalences.

Actually there is a specific based circle action on $B G$, that we also expect to be equivalent. A cyclic space is a contravariant functor from $\Lambda$ to the category of topological spaces, and in particular it has the underlying structure of a simplicial space. The realization of a cyclic space is equipped with a circle action [9]. We recall from [9] that the space $L B G$ is equivalent (by a $S^{1}$-equivariant map that is a non-equivariant equivalence) to the realization of a cyclic space $l b G$ with $(l b G)_{n}=G^{n+1}$, and

$$
\begin{aligned}
& d_{i}\left(x_{0}, \ldots, x_{n}\right)=\left(x_{0}, \ldots, x_{i-1}, x_{i} x_{i+1}, x_{i+2}, \ldots, x_{n}\right) \\
& s_{i}\left(x_{0}, \ldots, x_{n}\right)=\left(x_{0}, \ldots, x_{i-1}, e, x_{i+1}, x_{i+2}, \ldots, x_{n}\right) \\
& t_{n}\left(x_{0}, \ldots, x_{n}\right)=\left(x_{n}, x_{0}, x_{1}, \ldots, x_{n-1}\right) .
\end{aligned}
$$

Here $e$ is the unit of $G$. The realization $|l b G|$ of $l b G$ can be thought of as the space of finite configurations of points on the circle with summable labels in $G$ [21]. There is a cyclic subspace $b G \subset l b G$ that at level $n$ is the space $(b G)_{n}$ of $n$-tuples $\left(g_{0}, \ldots, g_{n}\right)$ such that the ordered product satisfies $g_{0} \cdots g_{n}=1$. The homeomorphism $(b G)_{n} \cong G^{n}$ induced forgetting $g_{0}$ gives an isomorphism between $b G$ and the classical simplicial construction $B_{*} G$ with realization $\left|B_{*} G\right|=B G$. The category $\Lambda$ is well known to be non-canonically isomorphic to its opposite $\Lambda^{o p}$. The choice of an isomorphism gives a bijective correspondence between cyclic spaces and cocyclic spaces. In particular under the isomorphism described in [9, Section 1] the cyclic space $b G$ corresponds to the cocyclic space $\omega G$ of Example 2.4.

Example 7.9 (String topology in the category of correspondences) The cocyclic space $l X$ does not come from a cyclic operad because of course the totalization $L X$ is not a double loop space in general. However if we work in the category Cor of correspondences this works. Objects in Cor are topological spaces and a morphisms $X \rightarrow Y$ is a pair of maps $X \stackrel{f}{\longleftarrow} Z \stackrel{g}{\longrightarrow} Y$. The composition is defined via pullbacks. When the backward map $f$ is an inclusion we can view a morphism as a map defined only partially. If $f$ is a homeomorphism then we identify the morphism to the honest map $g \circ f^{-1}: X \rightarrow Y$. The cartesian product induces a monoidal structure on Cor and it makes sense to consider operads in Cor. 
It turns out that the collection $l X$ forms a cyclic operad with multiplication in the category of correspondences.

The composition map

$$
\circ_{i}:(l X)_{m} \times(l X)_{n} \rightarrow l X_{m+n-1}
$$

is defined on each pair $\left(\left(x_{0}, \ldots, x_{m}\right),\left(y_{0}, \ldots, y_{n}\right)\right)$ such that $x_{i}=y_{0}$, sending it to

$$
\left(x_{0}, \ldots, x_{i-1}, y_{1}, \ldots, y_{n}, x_{i+1}, \ldots, x_{m}\right) \text {. }
$$

The operad map from the associative operad Ass $\rightarrow l X$ is defined at level $n$ by the correspondence $* \leftarrow X \stackrel{\Delta}{\rightarrow} X^{n+1}$, where $\Delta$ is the inclusion of the thin diagonal. The associated cosimplicial object is the honest cosimplicial space $l X$. A small variant of Theorem 7.7 shows that the totalization $L X=\operatorname{Tot}(l X)$ admits the action of the operad $f M S$ in the category of correspondences. This is exactly the action constructed by Sasha Voronov [25], at least on the suboperad Cacti $\subset f M S$, and that motivated him to work in the category of correspondences.

Further directions Kaufmann [12; 13] and Kontsevich-Soibelman [14] proved that the Hochschild complex of a Frobenius algebra has an action on the chain level of the operad $\mathcal{M}$ of moduli spaces of Riemann surfaces with boundary. The restriction of this action to the operad of genus 0 surfaces is the cyclic Deligne conjecture. The generalization works because the endomorphism operad of a Frobenius algebra is a modular operad [15] with multiplication. Let $O$ be a modular operad in based spaces. It is natural to ask whether the totalization $\operatorname{Tot}\left(O^{*}\right)$ admits an action of the operad $\mathcal{M}$.

\section{Appendix: comparison with the McClure-Smith construc- tion}

We give the proof of Proposition 4.6. More generally we produce for any $m$ a geometric construction of an operad $M S_{m}$ that is isomorphic to the $E_{m}$ operad called $\mathcal{D}_{m}$ by McClure-Smith in [18]. In this section $\mathcal{D}_{m}$ will denote such operad and not the little $m$-discs operad.

Definition 8.1 Let $M S_{m}(n)=\mathcal{F}_{m}(n) \times \operatorname{Mon}(I, \partial I)$, where $\mathcal{F}_{m}(n)$ is defined similarly as $\mathcal{F}(n)$ in Definition 4.1 except that the 4 -tuple $\left(z_{1}, z_{2}, z_{3}, z_{4}\right)$ is replaced by a $(m+2)$-tuple $\left(z_{1}, \ldots, z_{m+2}\right)$ with $z_{2 i-1} \in \stackrel{\circ}{I}_{j}(x)$ and $z_{2 i} \in \stackrel{\circ}{I}_{k}(x)$ for all $i$. The space $\mathcal{F}_{m}(n)$ is a regular CW-complex with a cell for each sequence assuming all values between 1 and $n$, with no equal adjacent values, and with no subsequence of 
length $m+1$ of the form $a b a b \ldots$, for $a \neq b$. The cell is identified to the product $\prod_{j=1}^{n} \Delta^{d(j)}$, where $d(j)$ is one less than the number of occurrences of $j$ in the sequence. Clearly $\mathcal{F}(n)=\mathcal{F}_{2}(n)$ and $M S=M S_{2}$.

The direct limits are denoted $M S_{\infty}=\lim _{m} M S_{m}$ and $\mathcal{F}_{\infty}(n)=\lim _{m} \mathcal{F}_{m}(n)$. There is a symmetric operad structure on $M S_{m}$ that is defined as for $M S$ in Section 4 by an embedding onto a suboperad of $\operatorname{Coend}\left(S^{1}\right)$.

Proposition 8.2 There is an isomorphism between the operad $M S_{\infty}$ and the $E_{\infty}$ operad $\mathcal{D}$ in [18]. This restricts for each $m$ to an isomorphism between $M S_{m}$ and the $E_{m}$ operad denoted $\mathcal{D}_{m}$ in [18].

Proof By definition $\mathcal{D}(k)=\operatorname{Tot}\left(Y_{k}^{*}\right)$, where $Y_{k}^{*}=\Xi_{k}\left(\Delta^{*}, \ldots, \Delta^{*}\right)$ is the cosimplicial space described in [18, Section 6]. Given cosimplicial spaces $X_{1}^{*}, \ldots, X_{k}^{*}$, the degree $s$ space $\Xi_{k}\left(X_{1}^{*}, \ldots, X_{k}^{*}\right)^{S}$ is the colimit of a functor $G_{1}: A_{1} \rightarrow$ Top to the category of topological spaces. Here $A_{1}$ is the category whose objects are pairs $(f, h)$ consisting of a monotone map $f:[t] \rightarrow[s]$ and a map $h:[t] \rightarrow\{1, \ldots, k\}$, for some $t$. A morphism from $(f, h)$ to $\left(f^{\prime}, h^{\prime}\right)$ is an ordered map $g:[t] \rightarrow\left[t^{\prime}\right]$ between the respective domains such that $f^{\prime} \circ g=f$ and $h^{\prime} \circ g=h$. The functor $G_{1}$ sends a pair $(f, h)$ to $\prod_{1 \leq i \leq k} X^{\left|f^{-1}(i)\right|-1}$.

In the special case $X_{1}^{*}=\cdots=X_{k}^{*}=\Delta^{*}\left[18\right.$, Corollary 12.3] states that $Y_{k}^{s}$ is a regular CW complex with a cell for each pair $(f, h)$ as above such that $f$ is surjective and for $0 \leq j<t$ either $f(j) \neq f(j+1)$ or $h(j) \neq h(j+1)$. Such cell has dimension $t+1-k$ and is $G_{1}(f, h)=\prod_{1 \leq i \leq k} \Delta^{\left|f^{-1}(i)\right|-1}$. Thus there is a natural identification between the cell of $Y_{k}^{0}$ indexed by $(f, *)$ and the cell of $\mathcal{F}_{\infty}(k)$ indexed by the sequence $f(0), \ldots, f(t)$. Here $*:[t] \rightarrow[0]$ is the unique map. These identifications are compatible with the inclusion of boundary cells and give a cellular $\Sigma_{k}$-equivariant homeomorphism $\alpha: \mathcal{F}_{\infty}(k) \cong Y_{k}^{0}$. In [18, Proposition 12.7] McClure-Smith construct an isomorphism of cosimplicial spaces $\omega: Y_{k}^{*} \cong \Delta^{*} \times Y_{k}^{0}$. We describe geometrically the homeomorphism

$$
\Phi=\omega^{-1} \circ\left(\Delta^{s} \times \alpha\right): \Delta^{s} \times \mathcal{F}_{\infty}(k) \cong Y_{k}^{s} .
$$

A partition $x \in \mathcal{F}_{\infty}(k)$ is a subdivision of $S^{1}$ into $k 1$-manifolds. An element of the simplex $z \in \Delta^{s}$ determines $s$ points on the circle and generically $s$ intervals that we call coarse intervals, inducing a subdivision of the 1-manifolds into intervals that we call fine intervals. We number by $0, \ldots, t$ in the counterclockwise ordering the fine intervals. Let $f:[t] \rightarrow\{1, \ldots, k\}$ be the map sending the index of a fine interval to the index of the 1-manifold containing it. Similarly let $h:[t] \rightarrow[s]$ be the map sending the index of a fine interval to the index of the coarse interval containing it. The cell of $\Phi(z, x) \in Y_{k}^{s}$ 
is indexed by the pair $(f, h)$. The point $\Phi(z, x)$ of the cell $\prod_{1 \leq i \leq k} \Delta^{\left|f^{-1}(i)\right|-1}$ is determined by the lengths of the fine intervals: the $i$ th component corresponds to the lengths of the fine intervals contained in the $i$ th 1-manifold, that sum up to 1 and thus determine an element of $\Delta^{\left|f^{-1}(i)\right|-1}$. The homeomorphisms $\Phi$ induce a $\Sigma_{k}$-equivariant homeomorphism

$$
\Psi: \mathcal{D}(k)=\operatorname{Tot}\left(Y_{k}^{*}\right) \cong \operatorname{Tot}\left(\Delta^{*}\right) \times \mathcal{F}_{\infty}(k)=M S_{\infty}(k) .
$$

Given $f \in \mathcal{D}(a)$ and $g \in \mathcal{D}(b)$, ie cosimplicial maps $f^{*}: \Delta^{*} \rightarrow \Xi_{a}\left(\Delta^{*}, \ldots, \Delta^{*}\right)=$ $Y_{a}^{*}$ and $g^{*}: \Delta^{*} \rightarrow \Xi_{b}\left(\Delta^{*}, \ldots, \Delta^{*}\right)=Y_{b}^{*}$, we recall that the operadic composition $f \circ_{i} g \in \mathcal{D}(a+b-1)$ is the cosimplicial map

$$
\begin{aligned}
& \Delta^{*} \stackrel{f^{*}}{\rightarrow} Y_{a}^{*} \stackrel{\Xi_{a}\left(\mathrm{id}, \ldots, g^{*}, \ldots, \mathrm{id}\right)}{\longrightarrow} \Xi_{a}\left(\Delta^{*}, \ldots, \Xi_{b}\left(\Delta^{*}, \ldots, \Delta^{*}\right), \ldots, \Delta^{*}\right) \\
& \quad \stackrel{\Gamma}{\cong} \Xi_{a+b-1}\left(\Delta^{*}, \ldots, \Delta^{*}\right)
\end{aligned}
$$

where $\Gamma$ is the cosimplicial isomorphism described in [18, Section 6]. Via the geometric correspondence $\Phi$ it is straightforward to check that for any $x \in \Delta^{s}$

$$
\Psi\left(f \circ_{i} g\right)(x)=\left[\Psi(f) \circ_{i} \Psi(g)\right](x) \in \Delta^{s} \times \mathcal{F}_{\infty}(a+b-1),
$$

and so $\Psi: \mathcal{D} \cong M S_{\infty}$ is an operad isomorphism.

For a fixed $m$ let $Z_{*}^{k} \subset Y_{*}^{k}$ be the cosimplicial subspace in section 13 of [18]. The $\Sigma_{k}$-equivariant homeomorphism $\alpha: \mathcal{F}_{\infty}(k) \cong Y_{0}^{k}$ restricts to a $\Sigma_{k}$-equivariant homeomorphism $\mathcal{F}_{m}(k) \cong Z_{0}^{k}$, because the cells of $\mathcal{F}_{m}(k)$ correspond to the cells of $Y_{0}^{k}$ whose indexing sequence has complexity not exceeding $m$, ie the cells of $Z_{0}^{k}$. The splitting $\omega: Y_{*}^{k} \cong \Delta^{*} \times Y_{k}^{0}$ restricts to a splitting $Z_{*}^{k} \cong \Delta^{*} \times Z_{k}^{0}$. The $E_{m}-$ operad $\mathcal{D}_{m}$ in [18] has as $k$ th space $\mathcal{D}_{m}(k)=\operatorname{Tot}\left(Z_{*}^{k}\right)$. Thus the operad isomorphism $\Psi: \mathcal{D} \cong M S_{\infty}$ restricts to an isomorphism between the suboperad $\mathcal{D}_{m} \subset \mathcal{D}$ in [18] and the suboperad $M S_{m} \subset M S$.

Proposition 4.6 follows as a special case for $m=2$.

\section{References}

[1] J M Boardman, R M Vogt, Homotopy invariant algebraic structures on topological spaces, Lecture Notes in Mathematics, Vol. 347, Springer, Berlin (1973) MR0420609

[2] R Budney, The operad of framed discs is cyclic, J. Pure Appl. Algebra 212 (2008) 193-196 MR2355044 
[3] R L Cohen, A A Voronov, Notes on string topology, from: "String topology and cyclic homology", Adv. Courses Math. CRM Barcelona, Birkhäuser, Basel (2006) 1-95 MR2240287

[4] V Drinfeld, On the notion of geometric realization, Mosc. Math. J. 4 (2004) 619-626, 782 MR2119142

[5] M Gerstenhaber, The cohomology structure of an associative ring, Ann. of Math. (2) 78 (1963) 267-288 MR0161898

[6] E Getzler, Batalin-Vilkovisky algebras and two-dimensional topological field theories, Comm. Math. Phys. 159 (1994) 265-285 MR1256989

[7] E Getzler, M M Kapranov, Cyclic operads and cyclic homology, from: "Geometry, topology, \& physics”, Conf. Proc. Lecture Notes Geom. Topology, IV, Int. Press, Cambridge, MA (1995) 167-201 MR1358617

[8] P Hu, The Hochschild cohomology of a Poincaré algebra arXiv:0707.4118

[9] J D S Jones, Cyclic homology and equivariant homology, Invent. Math. 87 (1987) 403-423 MR870737

[10] R Kaufmann, On several varieties of cacti and their relations, Algebr. Geom. Topol. 5 (2005) 237-300 MR2135554

[11] R Kaufmann, A proof of a cyclic version of Deligne's conjecture via cacti arXiv: math.QA/0403340

[12] R Kaufmann, Moduli space actions on the Hochschild co-chains of a Frobenius algebraI arXiv:math.AT/0606064

[13] R Kaufmann, Moduli space actions on the Hochschild co-chains of a Frobenius algebra II arXiv:math.AT/0606065

[14] M Kontsevich, Y Soibelman, Notes on A-infinity algebras, A-infinity categories and non-commutative geometry. I arXiv:math.RA/0606241

[15] M Markl, S Shnider, J Stasheff, Operads in algebra, topology and physics, Mathematical Surveys and Monographs 96, American Mathematical Society, Providence, RI (2002) MR1898414

[16] P May, The geometry of iterated loop spaces, Lectures Notes in Mathematics 271, Springer (1972)

[17] J E McClure, J H Smith, A solution of Deligne's Hochschild cohomology conjecture, from: "Recent progress in homotopy theory (Baltimore, MD, 2000)", Contemp. Math. 293, Amer. Math. Soc., Providence, RI (2002) 153-193 MR1890736

[18] J E McClure, J H Smith, Cosimplicial objects and little $n$-cubes. I, Amer. J. Math. 126 (2004) 1109-1153 MR2089084 
[19] J E McClure, J H Smith, Operads and cosimplicial objects: an introduction, from: "Axiomatic, enriched and motivic homotopy theory", NATO Sci. Ser. II Math. Phys. Chem. 131, Kluwer Acad. Publ., Dordrecht (2004) 133-171 MR2061854

[20] L Menichi, Batalin-Vilkovisky algebras and cyclic cohomology of Hopf algebras, $K$-Theory 32 (2004) 231-251 MR2114167

[21] P Salvatore, Configuration spaces with summable labels, from: "Cohomological methods in homotopy theory (Bellaterra, 1998)", Progr. Math. 196, Birkhäuser, Basel (2001) 375-395 MR1851264

[22] P Salvatore, Knots, operads, and double loop spaces, Int. Math. Res. Not. (2006) Art. ID 13628, 22 MR2276349

[23] P Salvatore, N Wahl, Framed discs operads and Batalin-Vilkovisky algebras, Q. J. Math. 54 (2003) 213-231 MR1989873

[24] DP Sinha, Operads and knot spaces, J. Amer. Math. Soc. 19 (2006) 461-486 MR2188133

[25] A A Voronov, Notes on universal algebra, from: "Graphs and patterns in mathematics and theoretical physics", Proc. Sympos. Pure Math. 73, Amer. Math. Soc. (2005) 81-103 MR2131012

Università di Roma Tor Vergata, Dipartimento di Matematica, Via della Ricerca Scientifica, Roma 00133, Italy,

salvator@mat.uniroma2.it

http://www.mat.uniroma2.it/ salvator/

Received: 25 June 2008 Revised: 5 November 2008 